\title{
A novel concept of measuring mass flow rates using flow induced stresses
}

\author{
P I JAGAD ${ }^{1, *}$, B P PURANIK ${ }^{2}$ and A W DATE ${ }^{2}$ \\ ${ }^{1}$ Department of Mechanical Engineering, Sinhgad College of Engineering, \\ Vadgaon (Bk), Pune 411 041, India \\ ${ }^{2}$ Department of Mechanical Engineering, Indian Institute of Technology Bombay, \\ Mumbai 400076, India \\ e-mail: jagadp@yahoo.com
}

MS received 8 March 2014; revised 28 December 2014; accepted 14 February 2015

\begin{abstract}
Measurement of mass flow rate is important for automatic control of the mass flow rate in many industries such as semiconductor manufacturing and chemical industry (for supply of catalyst to a reaction). In the present work, a new concept for direct measurement of mass flow rates which does not depend on the volumetric flow rate measurement and obviates the need for the knowledge of density is proposed from the measurement of the flow induced stresses in a substrate. The concept is formulated by establishing the relationship between the mass flow rate and the stress in the substrate. To this end, the flow field and the stress field in the substrate are evaluated simultaneously using a numerical procedure and the necessary correlations are derived. A least squares based procedure is used to derive the mass flow rate from the correlations as a function of the stress in the substrate.
\end{abstract}

Keywords. Channel flow; flow induced stress; fluid-blind; mass flow meter; maximum effective stress.

\section{Introduction}

A flow meter is used for automatic control of mass flow rate of a fluid in many industries. Basically, there are two types of mass flow meters: indirect and direct. In all indirect methods, mass flow rate $(\dot{m})$ is evaluated from measured volume flow rate $\dot{Q}\left(\mathrm{~m}^{3} / \mathrm{s}\right)$ as $\dot{m}(\mathrm{~kg} / \mathrm{s})=\dot{Q} \rho_{f}$, where $\rho_{f}\left(\mathrm{~kg} / \mathrm{m}^{3}\right)$ is the fluid density. Thus, while $\dot{Q}$ is estimated by measuring pressure drop $\triangle p$ across devices (venturi, orifice, etc.) of known dimensions, estimation of $\dot{m}$ can be made only through the knowledge of fluid density. During industrial operations, however, flowing fluids often undergo substantial variations of density due to changes in pressure, temperature, concentration, etc. In such situations, $\rho_{f}$ may not be known at all locations in the plant. Therefore, such a flow meter is not suitable for automatic control of mass flow rate.

*For correspondence 
Direct methods for measurement of $\dot{m}$ obviate the need for knowledge of $\rho_{f}$. There are mainly two types of direct mass flow meters: thermal mass flow meters and non-thermal mass flow meters. In the former (Huijsing et al 1988; Tison 1996; Viswanathan et al 2002; Figliola \& Beasley 2011), a known quantity of heat per unit time $(\dot{H})$ is supplied to the fluid while it passes through the meter and the increase in temperature $(\triangle T)$ of the fluid, under steady condition, is measured. Then the mass flow rate is computed using the relation $\dot{H}=\dot{m} c_{p} \triangle T$, where the fluid specific heat at constant pressure $\left(c_{p}\right)$ is assumed to be known. The thermal mass flow meters require calibration for each fluid medium and are not suitable if the fluid specific heat changes considerably with the heating. The non-thermal mass flow meters are usually of Coriolis type. The Coriolis mass flow meter (Doebelin 1990; Raszillier \& Durst 1991; Enoksson et al 1996; Figliola \& Beasley 2011) consists of a vibrating/oscillating (at known frequency $\omega$ ) bent tube. The fluid experiences a Coriolis acceleration while passing through the tube and hence exerts a force on the tube. This force constitutes a couple due to which the bent tube twists. The angle of twist $\theta$ is then measured. Now, the mass flow rate is evaluated from the expression $\theta=4 L d \omega \dot{m} / K_{s}$, where $L$ stands for the length of the limbs of the tube, $d$ stands for the distance between the two limbs of the tube and $K_{s}$ is the meter constant. Coriolis mass flow meters are complex in construction and are expensive.

The present work has been motivated by the fact that when a fluid passes through a channel embedded in a thin plate, stress is induced in the plate. The level of stress in the plate is expected to be dependent on the mass flow rate through the channel. Therefore, it should be possible to predict the mass flow rate from the measurement of the stress in the plate. The objective of the present work is to evaluate this possibility. To this end, to establish the relationship between the mass flow rate through the channel and the stress in the plate, numerical solutions of the flow field in the channel and the stress field in the plate are obtained simultaneously and necessary correlations are derived. A least squares based procedure is proposed for the prediction of the mass flow rate from the correlations. Thus, the proposed concept is formulated. The primary advantage of the proposed concept is that it is fluid-blind and does not need the knowledge of the fluid density. However, we note that it is not suitable for compressible flows.

\section{The proposed concept}

The schematic of the proposed device is shown in figure 1. It consists of a U-shaped channel embedded in a thin plate clamped on all external surfaces. The fluid to be measured is passed through this device. The stress in the plate is measured to predict the mass flow rate of the fluid.

\section{Formulation of the concept}

The concept is formulated by establishing a relationship between the mass flow rate through the channel and the stress in the plate. To this end, the flow field in the channel and the stress field in the plate are solved simultaneously using a numerical procedure (Jagad et al 2011) and functional relationships are established between the stress in the plate and the flow parameters. A least squares based procedure is employed to predict the mass flow rate. The details are as follows.

\subsection{Solution of flow and stress fields}

The governing equations and boundary conditions in dimensionless form are expressed as follows. 


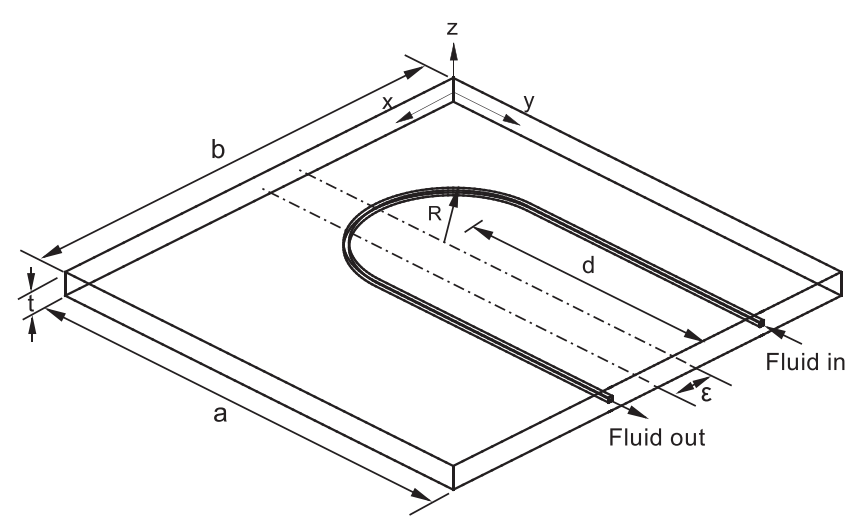

All external boundaries of the plate are assumed to be clamped

Figure 1. The proposed device for measuring mass flow rates.

3.1a Governing equations: The equations governing the transfer of mass and momentum for a fluid flow, in Cartesian coordinates and under steady state, can be written as

$$
\begin{gathered}
\frac{\partial u_{j}^{*}}{\partial x_{j}^{*}}=0, \\
\frac{\partial\left(u_{j}^{*} u_{i}^{*}\right)}{\partial x_{j}^{*}}=-\frac{\partial\left(p / \rho_{f} \bar{u}^{2}\right)}{\partial x_{i}^{*}}+\frac{1}{R e} \frac{\partial^{2} u_{i}^{*}}{\partial x_{j}^{*^{2}}},
\end{gathered}
$$

with usual notations. Here the hydraulic diameter $D_{h}$, the mean velocity $\bar{u}$, and the dynamic pressure $\rho_{f} \bar{u}^{2}$ are assumed to be the characteristic length, velocity and pressure scales respectively and the fluid properties are assumed to be constant.

Assuming no body forces, the equilibrium equation for an elastic solid can be written as (Jagad 2013)

$$
\frac{\partial\left(\sigma_{i j, s} / \rho_{f} \bar{u}^{2}\right)}{\partial x_{j}^{*}}=\frac{\partial}{\partial x_{j}^{*}}\left[\frac{\mu_{s}}{\rho_{f} \bar{u}^{2}}\left(\frac{\partial\left(\delta u_{i}^{*}\right)}{\partial x_{j}^{*}}+\frac{\partial\left(\delta u_{j}^{*}\right)}{\partial x_{i}^{*}}\right)+\frac{\lambda_{s}}{\rho_{f} \bar{u}^{2}} \frac{\partial\left(\delta u_{k}^{*}\right)}{\partial x_{k}^{*}} \delta_{i j}\right]=0,
$$

where $\sigma_{i j, s}$ stands for the stress tensor for the plate, $\delta u_{i}^{*}$ stands for the dimensionless displacement component in the direction - $i, \mu_{s}$ stands for the shear modulus, $\lambda_{s}$ stands for the Lame's constant, $\delta_{i j}$ stands for the Kronecker delta tensor, and $D_{h}$ is assumed to be the characteristic length scale as well as a reference scale for dimensionless representation of the displacement vector.

3.1b Interface condition: The condition of continuity of traction at the fluid-solid interface can be written as (Jagad 2013)

$$
-\left[\frac{p_{\text {out }}}{\rho_{f} \bar{u}^{2}}+(1-f) \frac{\Delta p}{\rho_{f} \bar{u}^{2}}\right] n_{i}+\frac{1}{R e}\left(\frac{\partial u_{i}^{*}}{\partial x_{j}^{*}}+\frac{\partial u_{j}^{*}}{\partial x_{i}^{*}}\right) n_{j}=\left(\frac{\sigma_{i j, s}}{\rho_{f} \bar{u}^{2}}\right) n_{j},
$$


where $p_{\text {out }}$ stands for the pressure at the channel outlet in excess of a reference pressure $p_{\text {ref }}$ that corresponds to zero stress condition, $f$ is a fraction whose value is a function of the distance of the point (along the interface) from the channel inlet/outlet, $\Delta p$ stands for the total pressure drop of the fluid in the channel, and $n_{j}$ stands for the outward facing unit normal vector for the channel walls.

The condition of continuity of velocity at the interface is redundant since the convection/velocity is assumed to be zero in the plate.

3.1c Boundary conditions: The boundary conditions for the fluid flow, in the dimensionless form, can be written as follows:

Assuming uniform velocity of the fluid at the channel inlet

$$
u_{i, B}^{*}=u_{i, i n}^{*},
$$

where the subscript $B$ stands for the boundary surface and $u_{i, i n}$ is the specified value of the velocity components at the inlet.

Assuming zero normal gradient of the flow variables at the channel outlet,

$$
\left(\frac{\partial u_{i}^{*}}{\partial n^{*}}\right)_{B}=0
$$

and

$$
\left(\frac{\partial p^{*}}{\partial n^{*}}\right)_{B}=0 .
$$

For the plate, the boundary conditions in dimensionless form can be written as follows:

Since external boundaries are assumed to be clamped, zero displacement vector is prescribed at the external boundaries of the plate

$$
\delta u_{i, B}^{*}=0 .
$$

Assuming a symmetry plane passing through the thickness of the plate, at the symmetry plane

$$
\delta u_{n, B}^{*}=0
$$

and

$$
\left[\frac{\partial\left(\delta u_{t}^{*}\right)}{\partial n^{*}}\right]_{B}=0,
$$

where $\delta u_{n}$ and $\delta u_{t}$ are the normal and tangential components of the displacement vector at a point on the symmetry plane respectively, and $\partial() / \partial n$ stands for the normal gradient.

From Eq. (4) it can be observed that there are three types of forces at the interface inducing stress in the plate. These are explained as follows. In the limit as fluid flow tends to zero, uniform pressure distribution equal to $p_{\text {out }} / \rho_{f} \bar{u}^{2}$ or $p_{\text {in }} / \rho_{f} \bar{u}^{2}$ (since $p_{\text {out }} \approx p_{\text {in }}$ in the limit as flow tends to zero) can be assumed throughout the channel. Thus, the stress can be assumed to be induced due to the static force alone acting on the channel wall due to $p_{\text {out }} / \rho_{f} \bar{u}^{2}$, and $\sigma_{i j, s} / \rho_{f} \bar{u}^{2}$ will be a function of $p_{\text {out }} / \rho_{f} \bar{u}^{2}$ alone. Now, with increasing value of the flow, a pressure distribution corresponding to $\triangle p / \rho_{f} \bar{u}^{2}$ is appended on the uniform pressure $p_{\text {out }} / \rho_{f} \bar{u}^{2}$. This pressure distribution is a function of $R e$. Now, there will be an additional stress induced in the plate due to the appended pressure distribution as well as due to the viscous 
stress $(1 / R e)\left(\partial u_{i}^{*} / \partial x_{j}^{*}+\partial u_{j}^{*} / \partial x_{i}^{*}\right)$. This additional stress will be a function of $R e$ as well as $p_{\text {out }} / \rho_{f} \bar{u}^{2}$. Furthermore, the dimensionless stress $\sigma_{i j, s} / \rho_{f} \bar{u}^{2}$ can be a function of the dimensionless elastic moduli $E / \rho_{f} \bar{u}^{2}$ and $v$ due to the application of the boundary conditions according to Eqs. (8) - (10). Thus, the dimensionless stress solution in the plate can be written in a functional form as

$$
\frac{\sigma_{i j, s}}{\rho_{f} \bar{u}^{2}}=f\left(\frac{E}{\rho_{f} \bar{u}^{2}}, v, \frac{p_{\text {out }}}{\rho_{f} \bar{u}^{2}}, \frac{\triangle p}{\rho_{f} \bar{u}^{2}}, R e\right) .
$$

From Eq. (2) (integrating this equation over the length of the channel) it can be observed that

$$
\frac{\Delta p}{\rho_{f} \bar{u}^{2}}=f_{1}\left(\operatorname{Re}, \frac{L}{D_{h}}\right) .
$$

Hence, the functional relationship in Eq. (11) can be rewritten as

$$
\frac{\sigma_{i j, s}}{\rho_{f} \bar{u}^{2}}=f_{2}\left(\frac{E}{\rho_{f} \bar{u}^{2}}, v, \frac{p_{\text {out }}}{\rho_{f} \bar{u}^{2}}, R e, \frac{L}{D_{h}}\right) .
$$

Additionally, the dependence of the dimensionless stress on dimensionless geometrical parameters shown in figure 1 emerge from the application of the boundary conditions. However, for a fixed geometry the functional relationship for a maximum effective stress in the plate can be written as

$$
\frac{\sigma_{e f f, \max }}{\rho_{f} \bar{u}^{2}}=f_{3}\left(\frac{E}{\rho_{f} \bar{u}^{2}}, v, \frac{p_{\text {out }}}{\rho_{f} \bar{u}^{2}}, R e\right) .
$$

where the effective stress or Von Mises stress is given by

$$
\sigma_{e f f}=\sqrt{0.5\left[\left(\sigma_{x x}-\sigma_{y y}\right)^{2}+\left(\sigma_{y y}-\sigma_{z z}\right)^{2}+\left(\sigma_{z z}-\sigma_{x x}\right)^{2}\right]+3\left(\sigma_{x y}^{2}+\sigma_{y z}^{2}+\sigma_{z x}^{2}\right)},
$$

with $\sigma_{x x}, \sigma_{x y}, \sigma_{x z}$, etc. standing for the components of the stress tensor $\sigma_{i j, s}$.

3.1d Parametric study: A parametric study is performed to evaluate the functional relationship in Eq. (14). The dimensions of the plate are assumed to be $a=b=50 \mathrm{~mm}, t=2.5 \mathrm{~mm}$. A square channel of cross-section $0.5 \mathrm{~mm} \times 0.5 \mathrm{~mm}$ is assumed to be placed symmetrically $(\varepsilon=0)$ in the plate with $R=10 \mathrm{~mm}$ and $d=30 \mathrm{~mm}$. The Poisson's ratio of $v=0.37$ is used assuming the plate material to be Polycarbonate. The effects of $R e, E / \rho_{f} \bar{u}^{2}$, and $p_{\text {out }} / \rho_{f} \bar{u}^{2}$ on $\sigma_{e f f, \text { max }} / \rho_{f} \bar{u}^{2}$ and that of $R e$ on $\triangle p / \rho_{f} \bar{u}^{2}$ are studied to derive the necessary correlations. The values of different parameters used for the study are summarized in table 1 . The Dean numbers (De), calculated using $D e=R e_{D_{h}} \sqrt{D_{h} / R_{c}}$ (Fellouah et al 2006) are such that the flow can be considered to be free of secondary flow structures. Depending on the aspect ratio, the curvature ratio, and the angular position in the channel, the critical Dean number varies from 80 to 500 (Fellouah et al 2006). The ranges of $E / \rho_{f} \bar{u}^{2}$ and $p_{\text {out }} / \rho_{f} \bar{u}^{2}$ employed for the study are fairly wide.

The symmetry of the problem in z-direction (along the thickness of the plate) is considered. The procedure developed in Jagad et al (2011) is employed for solving the problem. The mesh used is shown in figure 2. A hexahedral mesh is used in the channel and a hybrid mesh consisting of hexahedral and triangular prismatic cells is used in the plate. 
Table 1. Values of the parameters for the study.

\begin{tabular}{lc}
\hline Parameter & Values \\
\hline$R e$ & $292,380,468,585,877,1170,1460,1750,2050$ \\
$D e$ & $65,85,104.5,131,196,261.5,326.5,391.5,458.5$ \\
$E / \rho_{f} \bar{u}^{2}$ & $1.88 \times 10^{5}, 2.56 \times 10^{5}, 3.68 \times 10^{5}, 5.75 \times 10^{5}$, \\
$p_{\text {out }} / \rho_{f} \bar{u}^{2}$ & $1.02 \times 10^{6}, 2.3 \times 10^{6}, 9.2 \times 10^{6}$ \\
& $0,5,10,15,20,25,30,35,40,45,50,60,70,80$, \\
& $90,100,200,300,500,1000,2000,3000,4000,5000$ \\
\hline
\end{tabular}

Figure 3a shows the behavior of $\sigma_{e f f, \max } / \rho_{f} \bar{u}^{2}$ as a function of $R e$ for the values of $E / \rho_{f} \bar{u}^{2}$ varying from $1.88 \times 10^{5}$ to $9.2 \times 10^{6}$ and $p_{\text {out }} / \rho_{f} \bar{u}^{2}=0$. It can be observed that the plots collapse, meaning that $E / \rho_{f} \bar{u}^{2}$ does not affect $\sigma_{e f f, \max } / \rho_{f} \bar{u}^{2}$ at all. Moreover, this is verified for a large range of $p_{\text {out }} / \rho_{f} \bar{u}^{2}$ in figure $3 \mathrm{~b}$. The behavior of $\sigma_{e f f, \max } / \rho_{f} \bar{u}^{2}$ as a function of $p_{\text {out }} / \rho_{f} \bar{u}^{2}$ for the values of $R e$ varying from 292 to 2050 is shown in figure 4 . It is found that $\sigma_{e f f, \max } / \rho_{f} \bar{u}^{2}$ decreases with increase in $R e$ since the dimensionless pressure drop $\triangle p / \rho_{f} \bar{u}^{2}$ (see figure 5) and the dimensionless viscous stress $(1 / R e)\left(\partial u_{i}^{*} / \partial x_{j}^{*}+\partial u_{j}^{*} / \partial x_{i}^{*}\right)$ (see figure 6) at the channel wall decreases with increase in $R e$. Furthermore, the effect of $R e$ on $\sigma_{e f f, \text { max }} / \rho_{f} \bar{u}^{2}$ is found to be decreasing with increase in $p_{\text {out }} / \rho_{f} \bar{u}^{2}$. The percentage change in the values of $\sigma_{e f f, \max } / \rho_{f} \bar{u}^{2}$ with $R e$ varying from 292 to 2050 is found to vary from approximately $57 \%$ at $p_{\text {out }} / \rho_{f} \bar{u}^{2}=5$ to approximately $0.2 \%$ at $p_{\text {out }} / \rho_{f} \bar{u}^{2}=5000$. At low values of $p_{\text {out }} / \rho_{f} \bar{u}^{2}$ the pressure drop $\triangle p / \rho_{f} \bar{u}^{2}$ becomes dominant, and hence $R e$ has significant

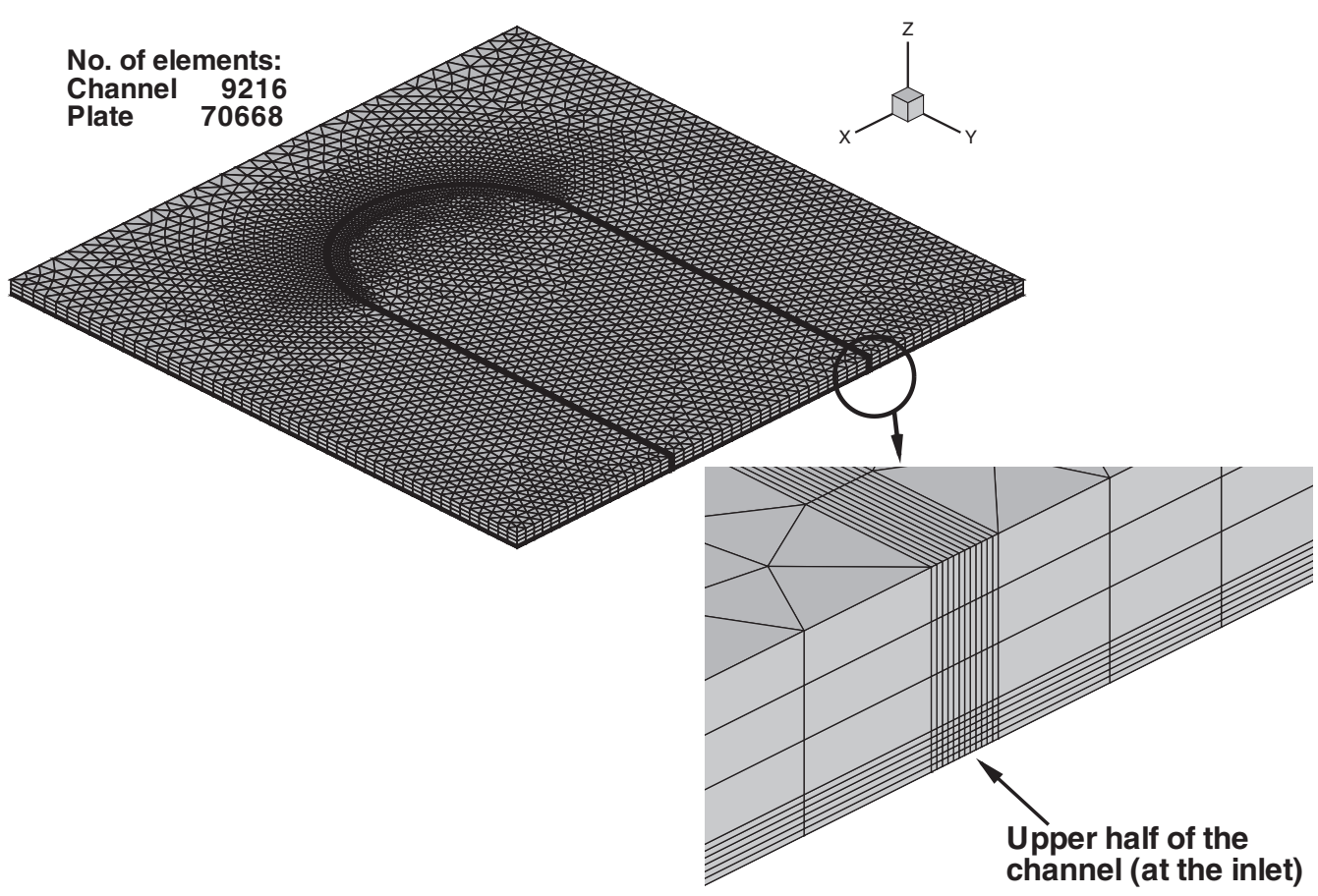

Figure 2. The mesh employed for the study. 


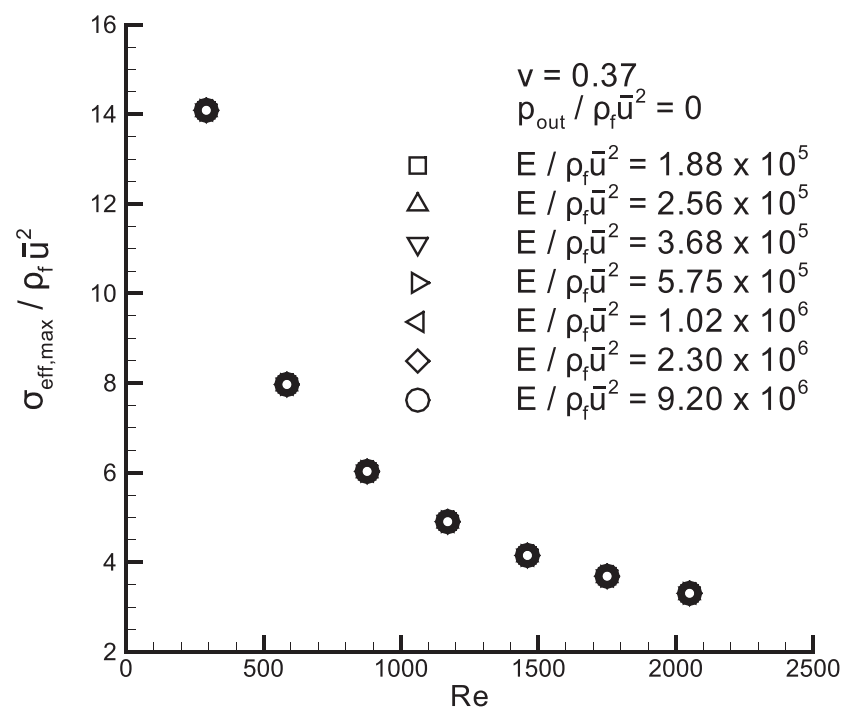

(a) $p_{\text {out }} / \rho_{\mathrm{f}} \bar{u}^{2}=0$

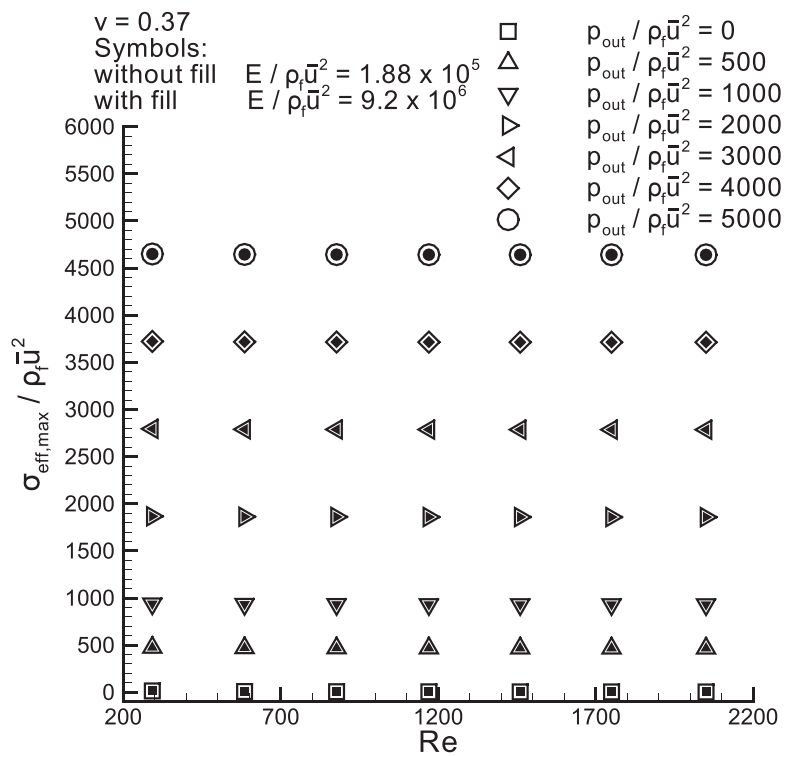

(b) A range of $p_{\text {out }} / \rho_{\mathrm{f}} \bar{u}^{2}$ from 0 to 5000

Figure 3. Behavior of $\sigma_{e f f, \max } / \rho_{f} \bar{u}^{2}$ as a function of $R e$ for different values of $E / \rho_{f} \bar{u}^{2}$, showing the redundancy of $E / \rho_{f} \bar{u}^{2}$.

effect on $\sigma_{e f f, \max } / \rho_{f} \bar{u}^{2}$ since $\triangle p / \rho_{f} \bar{u}^{2}$ is a function of Re. At high values of $p_{\text {out }} / \rho_{f} \bar{u}^{2}$ the fluid static pressure becomes the dominant factor and the effect of $\triangle p / \rho_{f} \bar{u}^{2}$ becomes insignificant. With increase in $p_{\text {out }} / \rho_{f} \bar{u}^{2}$, the pressure force on the channel walls increases and hence the dimensionless stress $\sigma_{e f f, \max } / \rho_{f} \bar{u}^{2}$ in the plate material increases. Since $\sigma_{e f f, \max } / \rho_{f} \bar{u}^{2}$ is 


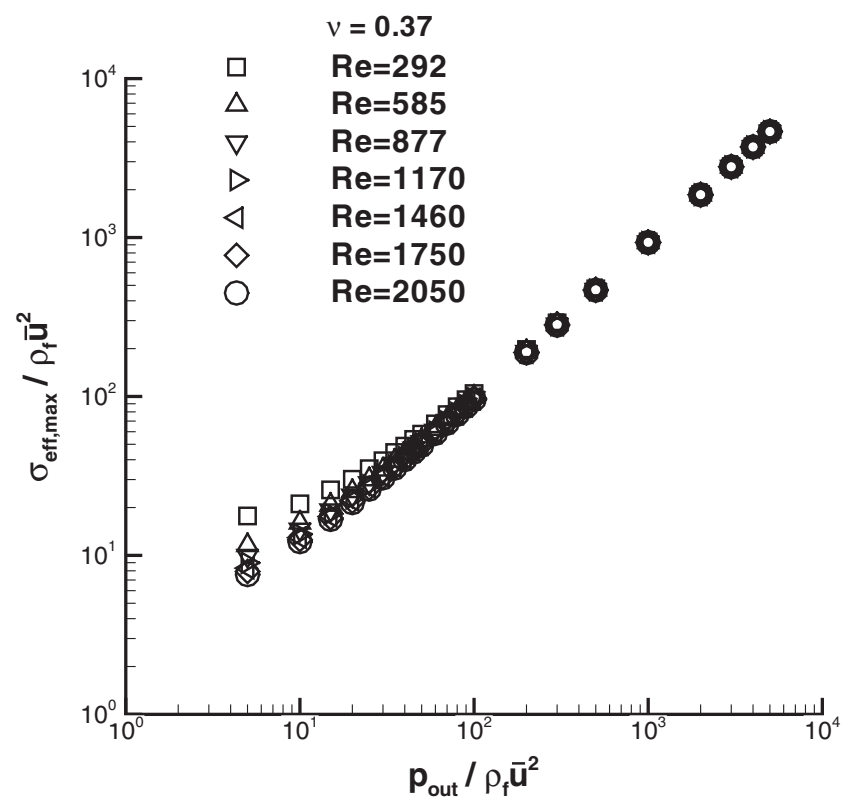

Figure 4. Behavior of $\sigma_{e f f, \max } / \rho_{f} \bar{u}^{2}$ as a function of $p_{\text {out }} / \rho_{f} \bar{u}^{2}$ for different values of $R e$.

found to be independent of $E / \rho_{f} \bar{u}^{2}$ and the value of $v$ is fixed, the relationship in Eq. (14) is now simplified as

$$
\frac{\sigma_{e f f, \max }}{\rho_{f} \bar{u}^{2}}=F_{4}\left(\operatorname{Re}, \frac{p_{\text {out }}}{\rho_{f} \bar{u}^{2}}\right) .
$$

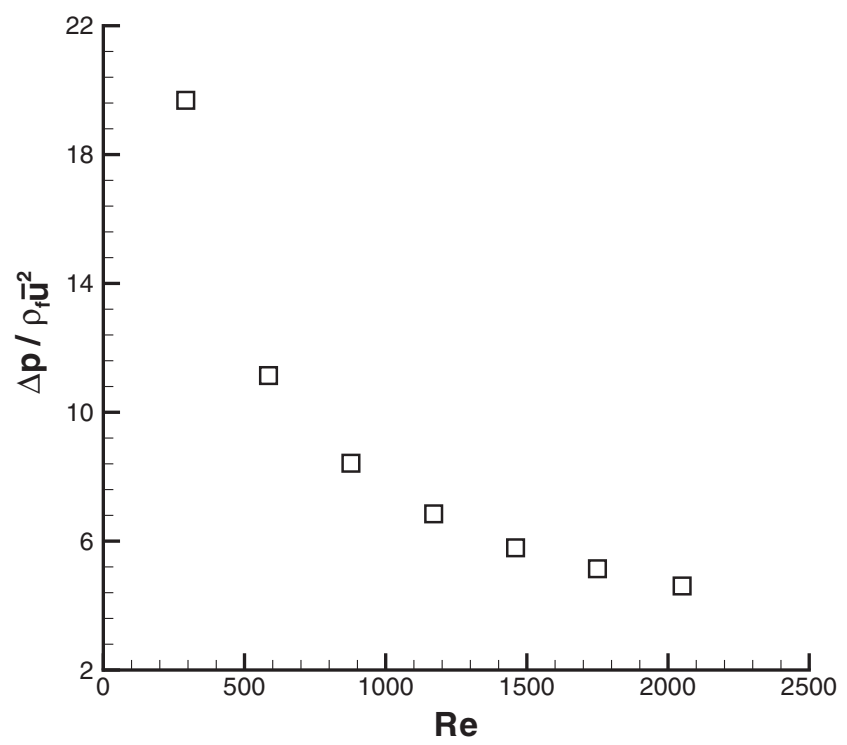

Figure 5. Behavior of $\Delta p / \rho_{f} \bar{u}^{2}$ as a function of $R e$. 


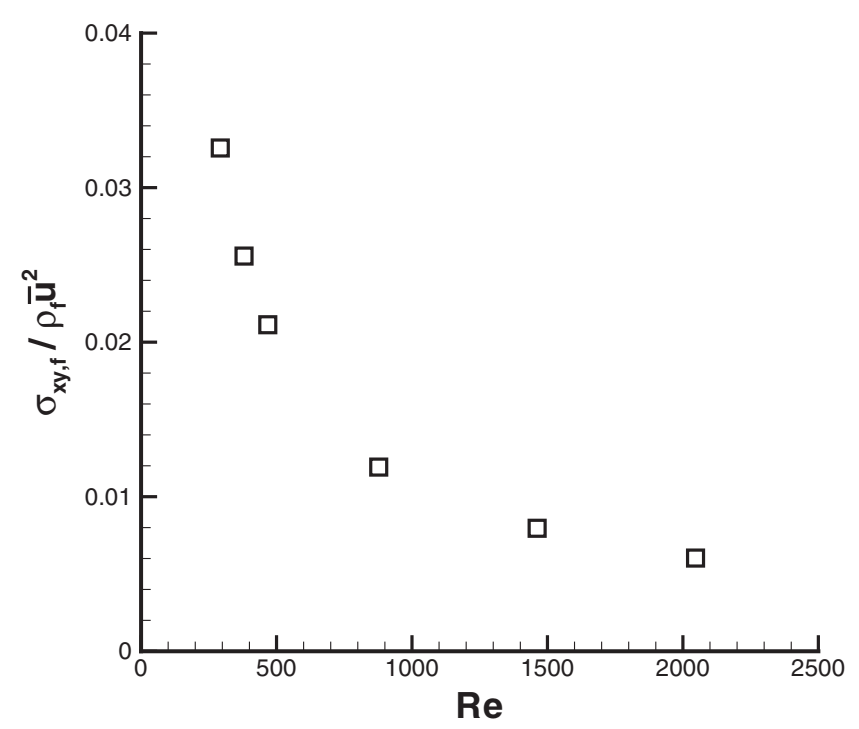

Figure 6. Behavior of $\sigma_{x y, f} / \rho_{f} \bar{u}^{2}$ (mean at the outer wall) as a function of $R e$.

Additionally, the point of location of $\sigma_{e f f, \max }$ is found to be at $x=15.8 \mathrm{~mm}, y=18 \mathrm{~mm}$, $z=0.1875 \mathrm{~mm}$ (in the vicinity of the end of the left limb of the channel) for all values of $R e$ when $p_{\text {out }} / \rho_{f} \bar{u}^{2}$ is greater than or equal to 15 , and for $R e$ greater than 877 when $p_{\text {out }} / \rho_{f} \bar{u}^{2}$ is less than 15 . For $p_{\text {out }} / \rho_{f} \bar{u}^{2}$ less than 15 and $R e$ less than 877 , this point is found to be located at $x=14.8 \mathrm{~mm}, y=49.5 \mathrm{~mm}, z=0.1875 \mathrm{~mm}$ (in the vicinity of the channel inlet). However, since the plate is thin, the variation of stress in $\mathrm{z}$-direction is very small and can be neglected.

\subsection{Development of correlations and the procedure}

As found in Section 3.1 (see Eq. (16)), the induced stress is a function of Re/ $\triangle p$ and $p_{\text {out }}$ for a given material of the plate (for a given $v$ ). Let $\sigma_{e f f, \max }, \Delta p$, and $p_{\text {out }}$ be measured. The maximum stress $\sigma_{e f f, \max }$ occurs at either of the two points depending on the values of $R e$ and $p_{\text {out }} / \rho_{f} \bar{u}^{2}$ as found in Section 3.1. These points are known a priori from the numerical analysis/ experiments. Strain gauges can be used to measure all components of the strain tensor and hence all stress components at these two points. Then, $\sigma_{\text {eff, } \max }$ can be computed according to Eq. (15) at these two points and the greater of the two values is regarded as the value of $\sigma_{e f f, \text { max }}$. A pressure gauge can be used to measure the fluid pressures (in excess of $p_{\text {ref }}$ ) at the channel inlet $\left(p_{\text {in }}\right)$ and outlet $\left(p_{\text {out }}\right)$ and the pressure drop calculated $(\triangle p)$. Otherwise, a manometer can be used to measure the pressure difference $(\triangle p)$ between the channel inlet and outlet and a pressure gauge can be used to measure $p_{\text {out }}$. Now the mass flow rate of the fluid can be derived as follows.

The total stress induced in the plate due to the passage of the flow through the channel can be written as

$$
\frac{\sigma_{e f f, \text { max }}}{\rho_{f} \bar{u}^{2}}=\frac{\sigma_{e f f, \text { max }, \text { static }}}{\rho_{f} \bar{u}^{2}}+\frac{\sigma_{e f f, \text { max }, \text { dynamic }}}{\rho_{f} \bar{u}^{2}},
$$

where the subscript "static" is used to represent the contribution due to $p_{\text {out }} / \rho_{f} \bar{u}^{2}$ alone in the limit as the flow tends to zero and the subscript "dynamic" is used to represent the additional 
contribution in the presence of the flow as has already been discussed (Section 3.1). From a few simulations (results not reported here) it is found that $\sigma_{e f f, \text { max static }} / \rho_{f} \bar{u}^{2}$ is a linear function of $p_{\text {out }} / \rho_{f} \bar{u}^{2}$. Furthermore, $\sigma_{e f f, \text { max }, \text { dynamic }} / \rho_{f} \bar{u}^{2}$ is assumed to be a function of $R e$ alone since its dependence on $p_{\text {out }} / \rho_{f} \bar{u}^{2}$ is found to be weak for a large range from the results (the dynamic stress is assumed to be decoupled from the static stress). Assuming a power law functional relationship for $\sigma_{e f f, \text { max,dynamic }} / \rho_{f} \bar{u}^{2}$, we can write

$$
\frac{\sigma_{e f f, \max }}{\rho_{f} \bar{u}^{2}}=c+m\left(\frac{p_{\text {out }}}{\rho_{f} \bar{u}^{2}}\right)+a_{1}(R e)^{b_{1}} .
$$

The following functional relationship is assumed for the fluid pressure drop

$$
\frac{\triangle p}{\rho_{f} \bar{u}^{2}}=a_{2}(R e)^{b_{2}}
$$

Using the numerical results reported in Section 3.1 and a regression analysis, the constants and powers in Eqs. (18) and (19) are evaluated, and hence the expressions are

$$
\frac{\sigma_{e f f, \text { max }}}{\rho_{f} \bar{u}^{2}}=0.928\left(\frac{p_{\text {out }}}{\rho_{f} \bar{u}^{2}}\right)+678.17(R e)^{-0.716},
$$

for $0 \leq p_{\text {out }} / \rho_{f} \bar{u}^{2} \leq 5000$, and $292 \leq R e \leq 2047$, and

$$
\frac{\triangle p}{\rho_{f} \bar{u}^{2}}=1284.31(R e)^{-0.74},
$$

for $292 \leq R e \leq 2047$.

The percentage error in reproducing the numerical results using Eqs. (20) and (21) is found to be as follows: $95 \%$ of the data points fall within $\pm 2 \%$ and $93 \%$ of the data points fall within $\pm 1 \%$ of the values predicted using Eq. (20). All data points fall within $\pm 3.2 \%$ of the values predicted using Eq. (21).

Now, a least squares based procedure can be employed to determine $\rho_{f}$ and $\bar{u}$ as follows. From Eqs. (18) (taking into account that $c=0$ ) and (19), the following two expressions for $R e$ can be derived

or

$$
R e=\left[\frac{1}{a_{1}}\left(\frac{\sigma_{\text {eff,max }}}{\rho_{f} \bar{u}^{2}}-m\left(\frac{p_{\text {out }}}{\rho_{f} \bar{u}^{2}}\right)\right)\right]^{1 / b_{1}}
$$

$$
\operatorname{Re}=\left(a_{1}\right)^{-1 / b_{1}}\left(\rho_{f}\right)^{-1 / b_{1}}\left(\bar{u}^{2}\right)^{-1 / b_{1}}\left(\sigma_{\text {eff,max }}-m p_{\text {out }}\right)^{1 / b_{1}},
$$

and

or

$$
\operatorname{Re}=\left[\frac{1}{a_{2}}\left(\frac{\Delta p}{\rho_{f} \bar{u}^{2}}\right)\right]^{1 / b_{2}}
$$

$$
R e=\left(a_{2}\right)^{-1 / b_{2}}\left(\rho_{f}\right)^{-1 / b_{2}}\left(\bar{u}^{2}\right)^{-1 / b_{2}}(\Delta p)^{1 / b_{2}} .
$$

Now, the objective is to find the values of $\rho_{f}$ and $\bar{u}^{2}$ such that the absolute difference IEq. (23) Eq. (25)| is minimum. Formally, this objective can be achieved as follows: A squared error can be defined as follows

$$
S=(\text { Eq. }(23)-\text { Eq. }(25))^{2}
$$


or

$$
\begin{gathered}
S=\left[\left(a_{1}\right)^{-1 / b_{1}}\left(\rho_{f}\right)^{-1 / b_{1}}\left(\bar{u}^{2}\right)^{-1 / b_{1}}\left(\sigma_{\text {eff,max }}-m p_{\text {out }}\right)^{1 / b_{1}}\right. \\
\left.-\left(a_{2}\right)^{-1 / b_{2}}\left(\rho_{f}\right)^{-1 / b_{2}}\left(\bar{u}^{2}\right)^{-1 / b_{2}}(\Delta p)^{1 / b_{2}}\right]^{2}
\end{gathered}
$$

Now our objective is to find the values of $\rho_{f}$ and $\bar{u}^{2}$ such that $S$ is minimum. This can be achieved by differentiating Eq. (27) with respect to $\rho_{f}$ and $\bar{u}^{2}$ and equating the derivatives to zero, to obtain

$$
\begin{gathered}
{\left[\left(a_{1}\right)^{-1 / b_{1}}\left(\rho_{f}\right)^{-1 / b_{1}}\left(\bar{u}^{2}\right)^{-1 / b_{1}}\left(\sigma_{\text {eff,max }}-m p_{\text {out }}\right)^{1 / b_{1}}-\left(a_{2}\right)^{-1 / b_{2}}\left(\rho_{f}\right)^{-1 / b_{2}}\left(\bar{u}^{2}\right)^{-1 / b_{2}}(\Delta p)^{1 / b_{2}}\right]} \\
{\left[\left(-1 / b_{1}\right)\left(a_{1}\right)^{-1 / b_{1}}\left(\rho_{f}\right)^{-1 / b_{1}-1}\left(\bar{u}^{2}\right)^{-1 / b_{1}}\left(\sigma_{\text {eff,max }}-m p_{\text {out }}\right)^{1 / b_{1}}\right.} \\
\left.-\left(-1 / b_{2}\right)\left(a_{2}\right)^{-1 / b_{2}}\left(\rho_{f}\right)^{-1 / b_{2}-1}\left(\bar{u}^{2}\right)^{-1 / b_{2}}(\triangle p)^{1 / b_{2}}\right]=0
\end{gathered}
$$

and

$$
\begin{gathered}
{\left[\left(a_{1}\right)^{-1 / b_{1}}\left(\rho_{f}\right)^{-1 / b_{1}}\left(\bar{u}^{2}\right)^{-1 / b_{1}}\left(\sigma_{\text {eff,max }}-m p_{\text {out }}\right)^{1 / b_{1}}-\left(a_{2}\right)^{-1 / b_{2}}\left(\rho_{f}\right)^{-1 / b_{2}}\left(\bar{u}^{2}\right)^{-1 / b_{2}}(\Delta p)^{1 / b_{2}}\right]} \\
{\left[\left(-1 / b_{1}\right)\left(a_{1}\right)^{-1 / b_{1}}\left(\rho_{f}\right)^{-1 / b_{1}}\left(\bar{u}^{2}\right)^{-1 / b_{1}-1}\left(\sigma_{\text {eff,max }}-m p_{\text {out }}\right)^{1 / b_{1}}\right.} \\
\left.-\left(-1 / b_{2}\right)\left(a_{2}\right)^{-1 / b_{2}}\left(\rho_{f}\right)^{-1 / b_{2}}\left(\bar{u}^{2}\right)^{-1 / b_{2}-1}(\triangle p)^{1 / b_{2}}\right]=0 .
\end{gathered}
$$

Equations (28) and (29) constitute a set of two non-linear equations in terms of $\rho_{f}$ and $\bar{u}^{2}$ as unknowns. These equations are solved using Newton's method to derive the values of $\rho_{f}$ and $\bar{u}^{2}$.

To demonstrate the use of the above procedure a numerical example is presented. Assuming the measured values of $\sigma_{\text {eff,max }}, p_{\text {out }}$ and $\triangle p$ to be $100000.0 \mathrm{~Pa}, 110140.0 \mathrm{~Pa}$ and $27390.4 \mathrm{~Pa}$ respectively, Eqs. (28) and (29) are solved. Using the Newton's method with initial guess $\rho_{f}=$ $840.0 \mathrm{~kg} / \mathrm{m}^{3}$ and $\bar{u}^{2}=4.1(\mathrm{~m} / \mathrm{s})^{2}$, the converged solution (which is assumed when the residuals have become less than $1.0 \times 10^{-5}$ (in 10 iterations)) is obtained to be $\rho_{f}=659.73 \mathrm{~kg} / \mathrm{m}^{3}$ and $\bar{u}^{2}=4.58(\mathrm{~m} / \mathrm{s})^{2}$. The channel cross section is assumed to be $2.5 \times 10^{-7} \mathrm{~m}^{2}$. Hence, the

Table 2. The range of mass flow rates that can be measured using the proposed concept for some common fluids.

\begin{tabular}{lcc}
\hline Fluid & Minimum $(\mathrm{kg} / \mathrm{s})$ & Maximum $(\mathrm{kg} / \mathrm{s})$ \\
\hline Air & $2.70 \times 10^{-06}$ & $1.89 \times 10^{-05}$ \\
Hydrogen & $1.31 \times 10^{-06}$ & $9.18 \times 10^{-06}$ \\
Helium & $2.91 \times 10^{-06}$ & $2.04 \times 10^{-05}$ \\
Water & $1.25 \times 10^{-04}$ & $8.76 \times 10^{-04}$ \\
Nitrogen & $2.52 \times 10^{-06}$ & $1.77 \times 10^{-05}$ \\
Oxygen & $3.03 \times 10^{-06}$ & $2.12 \times 10^{-05}$ \\
\hline
\end{tabular}


predicted / measured mass flow rate is $3.53 \times 10^{-4} \mathrm{~kg} / \mathrm{s}$. The ranges of mass flow rates that can be measured using this new concept (with the proposed size of the channel) for some common fluids are mentioned in table 2 .

\section{Conclusions}

A new concept of direct measurement of mass flow rates is presented. This concept is based on the measurement of the induced stress in a solid material through which the fluid flow to be metered is passed. The maximum value of the dimensionless effective stress $\left(\sigma_{e f f, \max } / \rho_{f} \bar{u}^{2}\right)$ is shown to be a function of the flow parameters such as the flow Reynolds number $(R e)$ or the dimensionless fluid pressure $\operatorname{drop}\left(\Delta p / \rho_{f} \bar{u}^{2}\right)$ and the dimensionless pressure of the fluid at the outlet $\left(p_{\text {out }} / \rho_{f} \bar{u}^{2}\right)$. A correlation is derived for the maximum value of the dimensionless effective stress and it is expressed in terms of the dimensionless flow parameters. It is shown that the measurement of $\sigma_{e f f, \max }, \Delta p$, and $p_{\text {out }}$ enables prediction of the mass flow rates. The present concept is simple and does not depend on a priori knowledge of the fluid properties for the measurement of mass flow rate.

\section{References}

Doebelin E O 1990 Measurement systems - Application and design. 4th edition, Mc-Graw-Hill Publishing Company, Singapore

Enoksson P, Stemme G and Stemme E 1996 A coriolis mass flow sensor structure in silicon. In: Micro electro mechanical systems, 1996, MEMS '96, Proceedings, pages 156-161

Fellouah H, Castelain C, Moctar A O E and Peerhossaini H 2006 A criterion for detection of the onset of dean instability in Newtonian fluids. European J. Mech. - B/Fluids 25(4): 505-531

Figliola R S and Beasley D E 2011 Theory and design for mechanical measurements. 5th edition, J. Wiley, Hoboken, NJ

Huijsing J H, Vandorp A L C and Loos P J G 1988 Thermal mass-flow meter. J. Phys. E-Scientific Instrum. 21(10): 994-997

Jagad P I 2013 An unstructured mesh finite volume methodology for conjugate heat transfer and fluidstructure interaction problems. $\mathrm{PhD}$ thesis, Indian Institute of Technology Bombay

Jagad P I, Puranik B P and Date A W 2011 A finite volume procedure on unstructured meshes for fluidstructure interaction problems. World Academy of Science, Engineering and Technology, Paris, France, pages 639-645

Raszillier H and Durst F 1991 Coriolis-effect in mass flow metering. Arch. Appl. Mech. 61: 192-214

Tison S A 1996 A critical evaluation of thermal mass flow meters. J. Vacuum Sci. Technol. A: Vacuum, Surfaces, Films 14(4): 2582-2591

Viswanathan M, Kandaswamy A, Sreekala S and Sajna K 2002 Development, modeling and certain investigations on thermal mass flow meters. Flow Measur. Instrum. 12(5-6): 353-360 